\title{
VARIATIONAL PRINCIPLES IN THE LINEAR THEORY OF VISCOELASTIC MEDIA WITH MICROSTRUCTURE*
}

\author{
$\mathrm{BY}$
}

\author{
KENNETH A. KLINE
}

Wayne State University

\begin{abstract}
1. Introduction. In 1963 Gurtin [1] gave variational principles for problems of both the creep and relaxation type in the linear quasi-static theory of inhomogeneous and anisotropic viscoelastic solids. His work unified and extended certain variational principles of classical elastostatics. In particular, Gurtin's first variational principle generalized the theorem of $\mathrm{Hu} \mathrm{Hai}-\mathrm{Chang}$ [2] and Washizu [3], while his second variational principle provided a generalization of the principle of stationary potential energy in elastostatics.
\end{abstract}

In this work we give variational principles which are analogous to the first and second of Gurtin's and which provide a unified development of the linear quasi-static theory of (inhomogeneous and anisotropic) viscoelastic solids with microstructure.

The subject of materials with microstructure has received considerable attention lately. As examples of recent investigations of theories of elastic materials with microstructure we cite the papers by Mindlin [4], Toupin [5], Suhubi and Eringen [6] and Fox [7]. Furthermore, Green, Naghdi and Rivlin [8] have developed a general theory of multipolar continuum mechanics in which alternative definitions of multipolar displacements are utilized. These recent researches are, of course, rooted to earlier works: for an excellent historical discussion see Toupin [5].

Our development of the theory of viscoelastic solids with microstructure will be guided by the work of Mindlin [4] and Toupin [5] on microstructure in elasticity, and will use the Stieltjes convolution. In this latter regard we follow the study of linear viscoelasticity theory by Gurtin and Sternberg [9].

Sec. 2 deals with certain kinematical aspects of theories of solids with microstructure. We record some results due to Toupin [5] and give his reduction of the basic kinematical measures for the nonlinear theory of microstructure in elasticity to the kinematical measures used in Mindlin's linear theory [4]. We also discuss the kinematical measures arising in the nonlinear theory of microstructure in viscoelasticity as given by DeSilva and Kline [10] and Kline [11], and, following [5], obtain appropriate measures for a linear theory of viscoelastic solids with microstructure.

In Sec. 3 we adopt and extend where necessary the definitions given by Gurtin [1] concerning variational principles in linear viscoelasticity. Sec. 4 is devoted to the derivation of variational principles appropriate to the linear quasi-static theory of viscoelastic solids with microstructure.

Before closing this section we would like to discuss briefly some variational theorems which have been developed for theories of couple stresses and generalized continua. ${ }^{\mathbf{1}}$

${ }^{*}$ Received December 2, 1968; revised version received March 7, 1969.

'I am most grateful to a referee who kindly provided some interesting references. 
The essential difference between these earlier works and the present paper is best described by considering the kinematics of deformation of continuous media with microstructure. In this paper we follow Ericksen and Truesdell [12] and Toupin [5] and associate a triad of directors with each material particle of the medium. The directors are intended to describe the microstructure of the continuum and are allowed to deform and rotate completely independently of the displacement field associated with the material particles. Theories involving less general concepts of deformable continua have been extensively developed in the literature and have provided interesting and important results. The common feature of such theories is that couple stresses arise naturally. However the notion of a double force (a symmetric second-order tensor whose presence was first deduced by Mindlin [4]) does not enter naturally unless the director triad is allowed to deform as well as rotate. The variational principles given here include the double force and appear to be the first general principles accounting for deformation and rotation of the directors.

Presently available variational principles, to which we now turn our attention, may be separated into two categories. Such classification is made not to imply degrees of utility or importance of the various variational theorems, but rather to facilitate discussion of the kinematical structure with which they are concerned. As examples of one class we cite the works of Koiter [13], Naghdi [14], Nowacki [15], and E. Reissner [16]. These authors consider a dynamic generalization of the classical theory of elasticity; they introduce couple stresses but relate the rotation field to the displacement gradients. Such theories do not, therefore, modify the kinematic structure of the continuous medium, but rather they retain the concept of a deformable continuum in the classical sense. However, a modification of the kinematic structure was not the purpose of these works, and the theories presented have been very useful in shell theory [12], [14], [16], and appear to provide insight for the study of elastic fatigue [13] and thermal stresses [15].

For representative papers dealing with a second class of variational theorems we refer the reader to the works of Günther [17], Nowacki [18] and E. Reissner and Wan [19]. These authors consider a rotation of the continuum which is independent of the displacement field. They are naturally led to the existence of a couple stress tensor which appears in the spin angular momentum balance equation. As Toupin [5] has pointed out, this spin momentum equation is actually the antisymmetric part of a more general balance equation. Toupin's comments (1964) concerning the symmetric part of this general balance equation are still valid. He said, in part: "The symmetric part . . . is not so easy to interpret in terms of familiar concepts." We term the symmetric part the microstructure stretch momentum balance equation and note it enters only when the microstructure is allowed to deform as well as rotate. The authors of [17], [18], [19] were not concerned with microstructure deformations; their work may be considered a refinement of the work discussed in the first class above. For example, in the recent work of E. Reissner and Wan [19] a variational principle is given which extends one obtained by Naghdi [14] and has, as the Euler equations, the linear and spin momentum equations, the strain displacement relations (which relate strain measures to the displacement and rotation fields), and the stress strain relations. The extension from [14] to [19] centers precisely on the kinematical structure assumed; as discussed above, Naghdi [14] considered a rotation which is related to the displacement field, while E. Reissner and Wan [19] considered rotations and displacements as independent quantities. In the sense that the present work considers rotations, displacements and micro- 
structure deformations as independent quantities, our results may be considered as further refinements. However, they are offered here in the broader hope they may shed light on the troublesome stretch momentum equation.

The notion that the microstructure can deform and rotate independently of material particle displacements is, of course, central to the work of Mindlin [4] and Toupin [5], and, in fact, the variational result most akin to ours was given by Mindlin. Mindlin obtained, from Hamilton's principle, the linear, spin and stretch momentum equations and the stress and couple stress boundary conditions. Our second variational principle may be considered as an extension of Mindlin's result to viscoelastic media with microstructure.

In our first variational principle we assume only that the double stress and the classical strain tensors are symmetric and obtain, as Euler equations, the linear, spin and stretch momentum equations, the full set of kinematical relations (which express strain measures in terms of the displacement, rotation and microstructure deformation fields), the constitutive equations for the stress, couple stress, and double force tensors (in terms of Stieltjes convolutions), the stress and couple stress boundary conditions, and the displacement and director difference boundary conditions. The restriction of symmetry for the double force and classical strain tensors does not, at this time, appear to be unreasonable, and in any event could be easily removed using the technique presented by E. Reissner [20].

Finally, as a possible application for the theory presented here we mention the problem of creeping motion (e.g. small shear rates) of a viscous fluid containing suspended deformable particles. Certain blood flows provide such an example since red cells are highly deformable. Following the imaginative work of Ericksen [21] some progress has been made in treating blood flow if the red cells are assumed rigid; one merely considers displacements and rotations as independent quantities and neglects microstructure deformations (see [22]). However, to account for the deformable character of red cells one must consider independent microstructure deformations, and hence deal with double forces and the stretch momentum equations. The present work provides a unified foundation for the necessary theory, and the variational formulation makes possible the application of well-known approximate solution techniques.

2. Preliminary remarks on solids with microstructure. We refer the motion of a continuous medium with microstructure to a single fixed rectangular Cartesian coordinate system and denote material points by $\mathrm{X}$. Following Toupin [5], the microstructure at time $t$ is described by the directors $\mathrm{d}_{\alpha}(\mathrm{X}, t), \alpha=1,2,3$. Thus the motion of a continuum with microstructure is represented by the mappings

$$
\mathbf{x}=\tilde{\mathbf{x}}(\mathbf{X}, t) \quad \mathbf{d}_{\alpha}=\tilde{\mathbf{d}}_{\alpha}(\mathbf{X}, t)
$$

where $\mathbf{x}$ is the position of $\mathbf{X}$ at time $t$.

Toupin [5] expressed the energy of deformation $W$ as a function of $\mathbf{d}_{\alpha}, \nabla \mathbf{x}, \nabla \mathbf{d}_{\alpha}$ and $\mathbf{X}$, where $\nabla=\partial / \partial \mathbf{X}$, and required $W$ to be invariant under the group of Euclidean displacements. From this he obtained the following kinematical measures appropriate for a nonlinear theory of elastic materials with microstructure:

$$
\begin{gathered}
\Delta_{\alpha K}=d_{\alpha i} x_{i, K}, \quad C_{K L}=x_{i, K} x_{i, L}, \\
\Delta_{\alpha L K}=d_{\alpha i, K} x_{i, L} .
\end{gathered}
$$

${ }^{2}$ An equivalent set of measures was given by Fox [7]. 
The theory of viscoelastic materials with microstructure was considered in [10] and [11]. In [11] constitutive equations were postulated in the form of functions of the kinematical variables

$$
\mathrm{d}_{\alpha}, \nabla \mathrm{x}, \quad \nabla \mathrm{d}_{\alpha}, \dot{\bar{\nabla} \mathrm{x}} \text { and } \dot{\overline{\nabla \mathrm{d}_{\alpha}}},
$$

where $(\cdot)$ indicates the material derivative ${ }^{3}$. Using the principle of material objectivity we obtained the following kinematical measures in terms of which a nonlinear theory of viscoelastic materials with microstructure could be formulated:

$$
\left\{\Delta_{\alpha K}, C_{K L}, \Delta_{\alpha L K}, \dot{C}_{K L}, V_{K L}, V_{K L M}\right\}
$$

where $\Delta_{\alpha K}, C_{K L}$ and $\Delta_{\alpha L K}$ are defined in (2.2) and

$$
\begin{aligned}
V_{K L} & =x_{i, K} x_{i, L}\left(v_{i, i}-d_{i}^{\alpha} \dot{d}_{\alpha i}\right), \\
V_{K L M} & =x_{i, K} x_{i, L} x_{k, m}\left(d_{i}^{\alpha} \dot{d}_{\alpha i}\right)_{, k} .
\end{aligned}
$$

In (2.4) $\mathbf{v}$ is the velocity, defined through (2.1) by $\mathbf{v}=\partial \tilde{\mathbf{x}} / \partial t$, and $\mathrm{d}^{\alpha}$ is the reciprocal to $\mathrm{d}_{\alpha}$. That is, we assume at each $\mathrm{X}$ the associated director triad forms a linearly independent set of vectors in the three-dimensional Euclidean space. A set of measures equivalent to (2.3) was given in [10].

Using the kinematical measures in (2.3) we wish to construct a linear theory. Let $\mathbf{D}_{\alpha}$ designate the director field at reference time $\tau$; i.e., say the functions in (2.1) satisfy

$$
\mathbf{X}=\tilde{\mathbf{x}}(\mathbf{X}, \tau), \quad \mathrm{D}_{\alpha}=\tilde{\mathrm{d}}_{\alpha}(\mathrm{X}, \tau) .
$$

Now recall that in obtaining infinitesimal strain measures for elastic materials with microstructure Toupin [5] formed the quantity $\gamma_{\alpha K}=D_{\alpha L} C_{L K}-\Delta_{\alpha K}$ and observed that $\gamma_{\alpha K}$ vanishes if each director is material, i.e., if the director field deforms and rotates along with the continuum. To obtain infinitesimal strain measures for viscoelastic materials with microstructure we first note from (2.4) that $V_{K L}$ vanishes if each director is material. This is immediately evident since if each director is material

$$
0=d_{\alpha i}-x_{i, K} D_{\alpha K} \text {. }
$$

Then taking a material derivative of (2.5) we obtain, using (2.5)

$$
0=\dot{d}_{\alpha i}-v_{i, i} x_{i, K} D_{\alpha K}=\dot{d}_{\alpha i}-v_{\imath, i} d_{\alpha i} .
$$

Multiplying this last equation by the inverse $d_{k}^{\alpha}$ yields that if (2.5) holds

$$
d_{k}^{\alpha} \dot{d}_{\alpha i}=v_{i, k} ; \quad V_{K L}=0 .
$$

Defining $E_{K L}$ by $C_{K L}=\delta_{K L}+2 E_{K L}$, it is clear that the basic set of kinematical measures (2.3) can be replaced without loss of generality by the set

$$
\left\{\gamma_{\alpha K}, E_{K L}, \Delta_{\alpha L K}, \dot{E}_{K L}, V_{K \bar{L}}, V_{K L M}\right\} .
$$

Let $\mathbf{u}$ be the displacement, defined by $\mathfrak{u}=\mathbf{x}-\mathbf{X}$, and, following [4] and [5], chonse $D_{\alpha L}=\delta_{\alpha L}$, and define

$$
\psi_{\alpha i}=d_{\alpha i}-D_{\alpha i}
$$

${ }^{3}$ Dependence on temperature and temperature gradients was also considered in [10] and [11] but is neglected here. 
We wish to obtain infinitesimal strain measures corresponding to the general measures in (2.6). Toupin [5] showed, to first-order terms in the displacement gradients $u_{i, i}$ and director differences $\psi_{\alpha_{i}}$, that

$$
\begin{aligned}
\gamma_{\alpha i}=u_{i, \alpha}-\psi_{\alpha i} ; \quad \Delta_{\alpha i j} & =\psi_{\alpha i, j}=\kappa_{\alpha i i} \\
E_{i j}=\frac{1}{2}\left(u_{i, i}+u_{i, i}\right) & =e_{i i} .
\end{aligned}
$$

It is easy to show, retaining first-order terms in $u_{i, j}, \partial u_{i, i} / \partial t, \psi_{\alpha i}, \partial \psi_{\alpha i} / \partial t, \psi_{\alpha_{i, i}}$ and $v_{i}$, that the infinitesimal strain measures corresponding to the last three measures in (2.6) have the form:

$$
\begin{gathered}
V_{i i}=\frac{\partial \gamma_{i j}}{\partial t} ; \quad V_{i j k}=\frac{\partial \psi_{i j, k}}{\partial t}=\frac{\partial \kappa_{i j k}}{\partial t} \\
\dot{E}_{i j}=\frac{\partial e_{i j}}{\partial t} .
\end{gathered}
$$

The quantities $\gamma_{i j}, \kappa_{i j k}$ and $e_{i j}$ are used in the linear theory of elasticity with microstructure as developed by Mindlin [4]. We see that additionally the measures $\partial \gamma_{i i} / \partial t$, $\partial \kappa_{i j k} / \partial t$ and $\partial e_{i j} / \partial t$ must enter to treat the linear theory of viscoelasticity with microstructure. Hence, for the theory of microstructure in viscoelastic solids, it is natural to postulate constitutive equations relating the stresses and double force to terms of the type $\boldsymbol{\gamma}^{*} d \mathbf{G}^{1}, \boldsymbol{r} * d \mathbf{G}^{2}$, and $\mathbf{e} * d \mathbf{G}^{3}$, where the $\mathbf{G}$ 's are various relaxation functions and the notation $f * d g$ stands for the Stieltjes convolution (see [9]).

3. Definitions. A certain economy is made possible in the statement of variational principles by first giving definitions of the problems, states and solutions one wants to consider. Such definitions are the core of the present section and are patterned after the systematic treatment given by Gurtin [1].

We consider an open, bounded, connected region of three-dimensional Euclidean space, $V$, with closure $\bar{V}$ and boundary $S$, where $S$ is the union of a finite number of nonintersecting closed regular surfaces (see Kellogg [23]). We allow $S$ to have a pair of complementary subsets $\left(S_{t}, S_{u}\right)$ and $\left(S_{\mu}, S_{\psi}\right)$, and denote the unit outward normal to $S$ by $\mathbf{n}$. Finally, we require the closure, e.g. $\bar{S}_{t}$, of each of the subsets of $S$ to be a regular surface.

The complete system of field equations in the linear quasi-static theory of viscoelastic solids with microstructure is required to hold on $V x(-\infty, \infty)$, the Cartesian product of region $V$ and the entire time interval, and can be expressed in the form

$$
\begin{gathered}
\gamma_{i j}=u_{i, i}-\psi_{i i}, \quad \kappa_{i j k}=\psi_{i j, k}, \\
e_{i j}=\frac{1}{2}\left(u_{i, i}+u_{j, i}\right), \\
t_{i i, i}+\rho f_{i}=0, \\
t_{j i}-m_{i i}+\mu_{k i i, k}+\rho b_{i i}=0 ; \quad m_{i j}=m_{i i}, \\
t_{i i}-m_{i i}=\gamma_{k n} * d B_{i i k n}-\left(\gamma_{k n}-e_{n k}\right) * d C_{i i k n}, \\
m_{i i}=2 e_{n k} * d A_{i i k n}+\left(\gamma_{k n}-e_{n k}\right) * d C_{i i k n}, \\
\mu_{k i i}=\kappa_{n a m} * d L_{k j i m n q} \text { on } V x(-\infty, \infty) .
\end{gathered}
$$

Eqs. (3.1) give the appropriate kinematical relations for solids with microstructure: 
strain measures $\gamma$ are defined in terms of displacement gradients and director differences $\Psi$; strain measures $\boldsymbol{k}$ are related to director difference gradients; and $\mathbf{e}$ is the classical infinitesimal strain tensor. Eq. (3.2) express the balance of linear momentum, with $\mathbf{t}$ the stress tensor and $\mathbf{f}$ the body force vector. The antisymmetric part of (3.3) gives the balance of spin momentum, while the symmetric part may be called the stretch momentum equation. Further, $\boldsymbol{m}$ is the double force tensor, $\boldsymbol{u}$ the double stress tensor, and tensor $\mathbf{b}$ arises since we allow the association of a body force vector $\mathbf{b}^{\alpha}$ with each director $\mathbf{d}_{\alpha}$; the antisymmetric part of $\mathbf{b}$ is a couple per unit mass.

In writing momentum equations (3.2) and (3.3) we have used the notation of Allen, DeSilva and Kline [24]. These governing equations were derived in [24] by postulating an energy balance equation and, following Green and Rivlin [25], by making use of invariance conditions under superposed rigid body motions. Similar governing equations were derived by Mindlin [4] and Toupin [5], both of whom used Hamilton's principle to obtain the variational equation of motion. For example, Mindlin's results (see Eqs. (4.1) of [4]) are the same as Eqs. (3.2) and (3.3) of the presen ${ }^{+}$work except for some notational differences: Mindlin's $\tau$ corresponds to our $\mathrm{m}$, his $\boldsymbol{\sigma}$ to our $\mathrm{t}-\mathrm{m}$, and his Ф to our b. Similarly one could relate symbols in Mindlin's theory to those used by Toupin or those used by the authors in [6], [7] and [8]. Here we only note that there are no standard notational agreements, perhaps due to certain conceptual differences among workers in this field. We compare explicitly to Mindlin's work to allow reference to his thorough discussion of constitutive equations (see Sec. 5 of [4]).

Constitutive relations for viscoelastic solids with microstructure are given in Eqs. (3.4), (3.5) and (3.6), where $\mathbf{A}, \mathbf{B}, \mathbf{C}$ and $\mathbf{L}$ are relaxation tensors. By the symmetry of $\mathbf{m}$ and inspection of (3.5) we are led to require

$$
A_{i i k m}=A_{i j k m}, \quad C_{i k m}=C_{i j k m} \quad \text { on } \bar{\Gamma} x(-\infty, 0) .
$$

Note that if A, B and $\mathbf{C}$ are isotropic tensors, then Eq.s. (3.4) and (3.5) are completely consistent with Mindlin's corresponding constitutive relations for $\boldsymbol{o}$ and $\tau$ (Eqs. (5.6), and (5.6) ${ }_{2}$ of [4]). In this case the constitutive relations for $\mathrm{t}-\mathrm{m}$ and $\mathrm{m}$ involve only seven independent relaxation functions.

We have already tacitly assumed that all quantities appearing in Eqs. (3.1) through (3.6) are defined on $V x(-\infty, \infty)$, and will now list additional requirements. We first adjoin to the field equations the initial conditions

$$
\begin{gathered}
\mathrm{u}=\boldsymbol{\psi}=\boldsymbol{\gamma}=\mathrm{e}=\boldsymbol{\kappa}=0, \\
\mathrm{~m}=\mathrm{t}=\mathfrak{u}=0 \quad \text { on } \quad \operatorname{Vx}(-\infty, \infty),
\end{gathered}
$$

and the mixed boundary conditions

$$
\begin{aligned}
t_{i}=t_{i i} n_{i}=\hat{t}_{i} \quad & \text { on } \quad S_{t} x(-\infty, \infty), \\
u_{i}=\hat{u}_{i} \quad & \text { on } \quad S_{u} x(-\infty, \infty), \\
\mu_{i i}=\mu_{k i i} n_{k}=\hat{\mu}_{i i} \quad & \text { on } \quad S_{\mu} x(-\infty, \infty), \\
\psi_{i i}=\hat{\psi}_{i j} \quad & \text { on } \quad S_{\psi} x(-\infty, \infty),
\end{aligned}
$$

where $\hat{\mathbf{t}}, \hat{\mathbf{u}}, \hat{\mathbf{u}}$ and $\hat{\mathbf{u}}$ are prescribed functions.

We now define a regular problem:

$$
R=R\left(V, S_{\mathfrak{t}}, S_{u}, S_{\mu}, S_{\psi}, \hat{\mathbf{t}}, \hat{\mathbf{u}}, \hat{\mathbf{u}}, \hat{\mathbf{\psi}}, \mathbf{f}, \mathbf{b}, \mathbf{A}, \mathbf{B}, \mathbf{C}, \mathbf{L}\right)
$$


is a regular problem for region $V$ with boundary $S$ having complementary subsets $\left(S_{t}, S_{u}\right),\left(S_{\mu}, S_{\psi}\right)$ as already defined if:

(i) $\hat{\mathbf{u}}$ and $\hat{\mathbf{\psi}}$ vanish on $\bar{S}_{u} x(-\infty, 0)$ and $\bar{S}_{\psi} x(-\infty, 0)$, respectively, and are continuous on $\bar{S}_{u} x[0, \infty)$ and $\bar{S}_{\psi} x[0, \infty)$, respectively;

(ii) $\hat{\mathrm{t}}$ and $\hat{\boldsymbol{u}}$ vanish on $\bar{S}_{t} x(-\infty, 0)$ and $\bar{S}_{\mu} x(-\infty, 0)$, respectively, and are piecewise continuous on $\bar{S}_{t} x[0, \infty)$ and $\bar{S}_{\mu} x[0, \infty)$, respectively, while $\hat{\mathbf{t}}(\mathbf{x}, \cdot)$ and $\hat{\mathbf{u}}(\mathbf{x}, \cdot)$ (the mappings of time alone) are continuous on $[0, \infty)$ for each $\mathbf{x} \in \bar{S}_{t}$ and for each $\mathbf{x} \in \bar{S}_{\mu}$, respectively;

(iii) $\mathbf{f}$ and $\mathbf{b}$ vanish on $\bar{V} x(-\infty, 0)$ and are continuous on $\bar{V} x[0, \infty)$;

(iv) $\mathbf{A}, \mathbf{B}, \mathbf{C}$ and $\mathrm{L}$ vanish on $\bar{V} x(-\infty, 0)$, are continuously differentiable on $\bar{V} x[0, \infty)$, and have the symmetry properties

$$
\begin{gathered}
A_{i j k m}=A_{k m i i}, \quad B_{i i k m}=B_{k m i i}, \quad C_{i i k m}=C_{k m i i}, \\
L_{k j i m n q}=L_{m n q k j i}, \quad \text { on } \bar{V} x(-\infty, \infty) .
\end{gathered}
$$

Further, $\mathbf{A}$ and $\mathbf{C}$ must satisfy the relations (3.7).

We note the symmetry relations given in (3.11) for the relaxation tensors A, B, C and $\mathbf{L}$ are of the same form as the symmetry relation assumed for the relaxation tensor in classical anisotropic viscoelasticity [1], and are identically satisfied if we reduce to the linear theory of microstructure in isotropic elastic materials [4].

Having specified the meaning of a regular problem, one's task is to find a set of functions

$$
\{\mathbf{u}, \boldsymbol{\psi}, \boldsymbol{\gamma}, \mathbf{e}, \boldsymbol{k}, \mathrm{m}, \mathbf{t}, \mathbf{u}\} \quad \text { on } \bar{V} x(-\infty, \infty)
$$

which satisfies the field equations (3.1) to (3.6), the initial conditions (3.8) and the boundary conditions (3.9). We seek an equivalent variational formulation for the preceding statement of the mixed boundary-value problem, and, to this end, now define an admissible state.

The ordered array of functions

$$
Q=[\mathbf{u}, \boldsymbol{u}, \boldsymbol{\gamma}, \mathbf{e}, \mathbf{k}, \mathbf{m}, \mathbf{t}, \mathbf{u}]
$$

is an admissible state on $\bar{V} x(-\infty, \infty)$ if:

(i) $\mathbf{u}, \boldsymbol{\psi}, \boldsymbol{\gamma}, \mathbf{e}, \boldsymbol{\kappa}, \mathbf{m}, \mathbf{t}$ and $\boldsymbol{u}$ vanish on $\bar{V} x(-\infty, 0)$ and are continuously differentiable on $\bar{V} x[0, \infty)$;

(ii) e and $\mathrm{m}$ satisfy the symmetry relations $e_{i i}=e_{i i}, m_{i j}=m_{j i}$ on $\bar{V} x(-\infty, \infty)$. Addition of states and multiplication of a state by a scalar are defined by

$$
\begin{aligned}
& a+\tilde{a}=[\mathbf{u}+\tilde{\mathbf{u}}, \boldsymbol{u}+\tilde{\mathbf{u}}, \boldsymbol{\gamma}+\tilde{\gamma}, \mathbf{e}+\tilde{\mathbf{e}}, \boldsymbol{k}+\tilde{\mathbf{k}}, \mathbf{m}+\tilde{\mathbf{m}}, \mathbf{t}+\tilde{\mathbf{t}}, \boldsymbol{u}+\tilde{\mathbf{u}}], \\
& \alpha Q=[\alpha \mathbf{u}, \alpha \boldsymbol{\psi}, \alpha \boldsymbol{\gamma}, \alpha \mathbf{e}, \alpha \boldsymbol{k}, \alpha \mathrm{m}, \alpha \mathbf{t}, \alpha \mathbf{u}],
\end{aligned}
$$

in which case the set of all admissible states is a linear space.

We now define a solution of the mixed boundary-value problem in terms of admissible states. Given a regular problem $R=R\left(V, S_{\iota}, S_{u}, S_{\mu}, S_{\psi}, \hat{\mathbf{t}}, \hat{\mathbf{u}}, \hat{\mathbf{u}}, \hat{\mathbf{u}}, \mathbf{f}, \mathbf{b}, \mathbf{A}, \mathbf{B}, \mathbf{C}, \mathbf{L}\right)$, we say $Q=[\mathbf{u}, \boldsymbol{u}, \boldsymbol{\gamma}, \mathbf{e}, \boldsymbol{\kappa}, \mathrm{m}, \mathbf{t}, \mathfrak{u}]$ is a regular solution of $R$ if $Q$ is an admissible state on $\bar{V} x(-\infty, \infty)$ which satisfies the field equations (3.1) to (3.6) and the boundary conditions (3.9).

By functional we mean a real-valued function defined on a subset of a linear space. 
If $L$ is a linear space, $\Omega\{\cdot\}$ a functional whose domain is $K, K \subseteq L$, then for

$$
a, \tilde{a} \in L, \quad a+\alpha \tilde{a} \in K \text { for every real } \alpha,
$$

we formally define the notation

$$
\delta_{a} \Omega\{Q\}=\left.\frac{d}{d \alpha} \Omega\{Q+\alpha \tilde{\mathscr{C}}\}\right|_{\alpha=0} .
$$

The variation of $\Omega\{\cdot\}$ is zero at $Q$ over $K$ and is written

$$
\delta \Omega\{a\}=0 \quad \text { over } K,
$$

if and only if $\delta_{\alpha} \Omega\{Q\}$ exists and equals zero for every choice of $\tilde{a}$ consistent with (3.14).

4. Variational principles characterizing viscoelastic solids with microstructure. In this section we consider two variational principles, the first of which is the more general since it treats admissible states which are not required to meet any of the field equations or boundary conditions. It should be noted from the definition that an admissible state (and hence a regular solution) is allowed to have finite jump discontinuities at time zero.

We begin by generalizing to solids with microstructure Gurtin's first variational principle [1], which in turn provided a generalization of the theorem due to $\mathrm{Hu} \mathrm{Hai}-$ Chang [2] and Washizu [3].

First variational principle. Let $K$ be the set of all admissible states on $\bar{V} x(-\infty, \infty)$. Let $R=R\left(V, S_{t}, S_{u}, S_{\mu}, S_{\psi}, \hat{\mathrm{t}}, \hat{\mathbf{u}}, \hat{\mathbf{u}}, \hat{\boldsymbol{\psi}}, \mathbf{f}, \mathrm{b}, \mathrm{A}, \mathrm{B}, \mathrm{C}, \mathrm{L}\right)$ be a regular problem. Let $a=[\mathfrak{u}, \mathfrak{u}, \boldsymbol{\gamma}, \mathbf{e}, \boldsymbol{k}, \mathrm{m}, \mathrm{t}, \mathfrak{u}] \in K$, and for each fixed $t \in(-\infty, \infty)$ define the functional $\Omega_{t}\{\cdot\}$ on $K$ by

$$
\begin{aligned}
& \Omega_{t}\{a\}=\int_{S_{v}} t_{i} * d \hat{u}_{i} d S+\int_{S_{t}}\left(t_{i}-\hat{t}_{i}\right) * d u_{i} d S \\
& +\int_{S \downarrow} \mu_{i j} * d \hat{\psi}_{i j} d S+\int_{S_{\mu}}\left(\mu_{i j}-\hat{\mu}_{i j}\right) * d \psi_{i j} d S \\
& -\int_{V}\left\{\left(t_{i i, i}+\rho f_{i}\right) * d u_{i}+\left(t_{i i}-m_{i i}+\mu_{k i i, k}+\rho b_{j i}\right) * d \psi_{j i}\right\} d V \\
& +\int_{V}\left\{\frac{1}{2} L_{k j i m n Q} * d \kappa_{n q m} * d \kappa_{j i k}-\frac{1}{2} C_{i i k n} * d\left(\gamma_{k n}-e_{k n}\right) * d\left(\gamma_{i i}-e_{j i}\right)\right. \\
& \left.+\frac{1}{2} B_{i i k n} * d \gamma_{k n} * d \gamma_{i i}+A_{i i k n} * d e_{k n} * d e_{i i}\right\} d V \\
& -\int_{V}\left\{\mu_{k j i} * d \kappa_{j i k}+\left(t_{i i}-m_{j i}\right) * d \gamma_{j i}+m_{i i} * d e_{i i}\right\} d V \text {. }
\end{aligned}
$$

Then

$$
\delta \Omega_{t}\{Q\}=0 \quad \text { over } K, t \in(-\infty, \infty)
$$

if and only if $a$ is a regular solution of $R$.

Proof. Let $\tilde{\boldsymbol{Q}}=[\tilde{\mathbf{u}}, \tilde{\mathbf{u}}, \tilde{\boldsymbol{\gamma}}, \tilde{\mathbf{e}}, \tilde{\mathbf{k}}, \tilde{\mathbf{m}}, \tilde{\mathbf{t}}, \tilde{\mathfrak{u}}] \in K$, from which it follows that $Q+\alpha \tilde{e} \in K$ for every scalar $\alpha$. Then by (4.1), (3.15), (3.11), the divergence theorem, property (ii) of admissible states, and Theorems 1.2 and 1.6 of [9],

$$
\delta_{a} \Omega_{b}\{Q\}=\int_{S_{a}}\left(\hat{u}_{i}-u_{i}\right) * d t_{i} d S+\int_{S_{\downarrow}}\left(\hat{\psi}_{i j}-\psi_{i i}\right) * d \tilde{\mu}_{i i} d S
$$




$$
\begin{aligned}
& +\int_{S_{i}}\left(t_{i j}-\hat{t}_{i}\right) * d \tilde{u}_{i} d S+\int_{S_{q}}\left(\mu_{i j}-\hat{\mu}_{i j}\right) * d \tilde{\psi}_{i j} d S \\
& +\int_{V}\left\{\left(\kappa_{n q m} * d L_{k i i m n q}-\mu_{k j i}\right) * d \tilde{\kappa}_{i i k}\right. \\
& +\left(2 e_{n k} * d A_{j i k n}+\left(\gamma_{k n}-e_{n k}\right) * d C_{i k k n}-m_{j i}\right) * d \tilde{e}_{j i} \\
& +\left(\gamma_{k n} * d B_{i i k n}-\left(\gamma_{k n}-e_{n k}\right) * d C_{i j k n}-\left(t_{i i}-m_{i i}\right)\right) * d \tilde{\gamma}_{i j} \\
& -\left(t_{i i, i}+\rho f_{i}\right) * d \tilde{u}_{i}-\left(t_{i i}-m_{j i}+\mu_{k i i, k}+\rho b_{i i}\right) * d \tilde{\psi}_{j i} \\
& -\left(\kappa_{i i k}-\psi_{i, k}\right) * d \tilde{\mu}_{k i i}-\left(e_{i i}-\frac{1}{2}\left(u_{i, i}+u_{i, i}\right)\right) * d \tilde{m}_{j i} \\
& \left.-\left(\gamma_{i i}-\left(u_{i, i}-\psi_{i i}\right)\right) * d\left(\tilde{t}_{j i}-\widetilde{m}_{j i}\right)\right\} d V, \quad(-\infty<t<\infty) \text {. }
\end{aligned}
$$

Clearly, if $a$ is a regular solution of $R$, then by virtue of (3.1)-(3.6) and (3.9), (4.3) yields

$$
\delta_{\tilde{a}}^{\tilde{a}} \Omega_{t}\{Q\}=0 \quad(-\infty<t<\infty) \text { for every } \tilde{a} \in K,
$$

which implies (4.2).

Turning to the converse assertion we need show that $a \in K$ is a regular solution of $R$ whenever (4.4) holds. Note that each of the integrands in (4.3) is a function of $(\mathrm{x}, t)$ and choose

$$
\begin{aligned}
\tilde{\mathbf{u}}(\mathbf{x}, t) & =\mathbf{u}^{\prime}(\mathbf{x}) h(t), & \tilde{\mathbf{q}}(\mathbf{x}, t) & =\mathbf{\psi}^{\prime}(\mathbf{x}) h(t), \\
\tilde{\boldsymbol{\gamma}}(\mathbf{x}, t) & =\mathbf{\gamma}^{\prime}(\mathbf{x}) h(t), & \tilde{\mathbf{e}}(\mathbf{x}, t) & =\mathbf{e}^{\prime}(\mathbf{x}) h(t), \\
\tilde{\mathbf{k}}(\mathbf{x}, t) & =\mathbf{x}^{\prime}(\mathbf{x}) h(t), & \tilde{\mathbf{m}}(\mathbf{x}, t) & =\mathbf{m}^{\prime}(\mathbf{x}) h(t), \\
\tilde{\mathbf{t}}(\mathbf{x}, t) & =\mathbf{t}^{\prime}(\mathbf{x}) h(t), & \tilde{\mathbf{u}}(\mathbf{x}, t) & =\mathbf{u}^{\prime}(\mathbf{x}) h(t)
\end{aligned}
$$

for every $(\mathbf{x}, t) \in \bar{V} x(-\infty, \infty)$, where $h$, the Heaviside unit step function, is defined by $h(t)=0 \quad(-\infty<t<0), h(t)=1 \quad(0 \leq t<\infty)$. Then, using (4.3), (4.5) and Theorem 1.2 in [9], we obtain from (4.4)

$$
\begin{aligned}
& \int_{S_{v}}\left(\hat{u}_{i}-u_{i}\right) t_{i j}^{\prime} n_{j} d S+\int_{S_{\downarrow}}\left(\hat{\psi}_{i j}-\psi_{i i}\right) \mu_{k i}^{\prime} n_{k} d S \\
& +\int_{S_{t}}\left(t_{1}-\hat{t}_{i}\right) u_{i}^{\prime} d S+\int_{S_{\mu}}\left(\mu_{i j}-\hat{\mu}_{i j}\right) \psi_{i j}^{\prime} d S \\
& +\int_{V}\left\{\left(\kappa_{n q m} * d L_{k i i m n Q}-\mu_{k i i}\right) \kappa_{i i k}^{\prime}\right. \\
& +\left(2 e_{n k} * d A_{i i k n}+\left(\gamma_{k n}-e_{n k}\right) * d C_{i \boldsymbol{i} k n}-m_{i \boldsymbol{i}}\right) e_{i \boldsymbol{i}}^{\prime} \\
& +\left(\gamma_{k n} * d B_{i k k n}-\left(\gamma_{k n}-e_{n k}\right) * d C_{i i k n}-\left(t_{i i}-m_{j i}\right)\right) \gamma_{j i}^{\prime} \\
& -\left(t_{i i, i}+\rho f_{i}\right) u_{i}^{\prime}-\left(t_{j i}-m_{i i}+\mu_{k i i, k}+\rho b_{j i}\right) \psi_{j i}^{\prime} \\
& -\left(\kappa_{j i k}-\psi_{i, k}\right) \mu_{k i i}^{\prime}-\left(e_{j i}-\frac{1}{2}\left(u_{i, j}+u_{j, i}\right)\right) m_{j i}^{\prime} \\
& \left.-\left(\gamma_{i i}-\left(u_{i, j}-\psi_{i i}\right)\right)\left(t_{i i}^{\prime}-m_{j i}^{\prime}\right)\right\} d V=0 \quad(-\infty<t<\infty) \text {. }
\end{aligned}
$$

Now (4.6) must hold for every $\mathbf{u}^{\prime}, \mathbf{u}^{\prime}, \boldsymbol{\gamma}^{\prime}, \mathbf{e}^{\prime}, \boldsymbol{k}^{\prime}, \mathrm{m}^{\prime}, \mathrm{t}^{\prime}, \boldsymbol{u}^{\prime}$ continuously differentiable on $\bar{V}$ with $\mathbf{e}^{\prime}$ and $\mathrm{m}^{\prime}$ symmetric. From this fact, the fundamental lemma of the calculus 
of variations, and the symmetries of $\mathbf{e}, \mathbf{m}, \mathbf{A}, \mathbf{C}$ it follows that $Q$ satisfies (3.1)-(3.6), $\left(3.9_{1}\right),\left(3.9_{3}\right)$ and

$$
\int_{S_{u}}\left(\hat{u}_{i}-u_{i}\right) t_{i i}^{\prime} n_{i} d S+\int_{S_{\psi}}\left(\hat{\psi}_{i j}-\psi_{i j}\right) \mu_{k j i}^{\prime} n_{k} d S=0 \quad \text { on } \quad(-\infty<t<\infty) \text {. }
$$

We need only confirm $\left(3.9_{2}\right)$ and $\left(3.9_{4}\right)$. Let $f$ and $g$ be functions continuously differentiable on $\bar{V}$, say $m, p, n, q, t$ are fixed integers $(m, p, n, q, t=1,2,3)$ and consider

$$
\begin{aligned}
t_{j i}^{\prime}(\mathbf{x}) & =\delta_{j m} \delta_{i p} g(\mathbf{x}), \\
\mu_{k i i}^{\prime}(\mathbf{x}) & =\delta_{k n} \delta_{i q} \delta_{i \ell} f(\mathbf{x}), \quad \mathbf{x} \in \bar{V} .
\end{aligned}
$$

Since (4.7) holds for every such $\mathbf{t}^{\prime}$ and $\boldsymbol{u}^{\prime}$ we obtain

$$
\left[\hat{u}_{p}(\mathbf{x}, t)-u_{p}(\mathbf{x}, t)\right] n_{m}(\mathbf{x})=0
$$

for every $(\mathbf{x}, t) \in S_{u} x(-\infty, \infty)$ with $\mathbf{x}$ a regular point, and

$$
\left[\hat{\psi}_{t q}(\mathbf{x}, t)-\psi_{t a}(\mathbf{x}, t)\right] n_{n}(\mathbf{x})=0
$$

for every $(\mathbf{x}, t) \in S_{\psi} x(-\infty, \infty)$ with $\mathbf{x}$ a regular point. Consider (4.10), fix (x,t) and choose coordinates such that $n_{n}(\mathbf{x}) \neq 0$ to conclude that

$$
\hat{\mathbf{\psi}}(\mathbf{x}, t)=\mathbf{\psi}(\mathbf{x}, t) \text { for every }(\mathbf{x}, t) \in S_{\psi} x(-\infty, \infty)
$$

with $\mathbf{x}$ regular. Hence from this and the continuity of $\boldsymbol{\psi}$ and $\hat{\boldsymbol{\psi}}$ we see $\left(3.9_{4}\right)$ holds. Similarly consider (4.9), use the continuity of $\mathbf{u}$ and $\hat{\mathbf{u}}$ to conclude $\left(3.9_{2}\right)$ holds and finally that $Q$ is a regular solution of $R$. This completes the proof.

To develop another form of variational principle we consider more stringent conditions on the admissible states. Define a kinematically admissible state as an admissible state which meets the kinematical relations (3.1), the constitutive relations (3.4)-(3.6) and the displacement and director difference boundary conditions $\left(3.9_{2}\right),\left(3.9_{4}\right)$. Such considerations lead to the following generalization of the theorem of minimum potential energy.

Second variational principle. Let $K$ be the set of all kinematically admissible states on $\bar{V} x(-\infty, \infty)$. Let $R=R\left(V, S_{t}, S_{u}, S_{\mu}, S_{\psi}, \hat{\mathbf{t}}, \hat{\mathbf{u}}, \hat{\mathbf{u}}, \hat{\mathbf{\psi}}, \mathbf{f}, \mathbf{b}, \mathbf{A}, \mathbf{B}, \mathbf{C}, \mathbf{L}\right)$ be a regular problem. Let $Q=[\mathbf{u}, \boldsymbol{\psi}, \boldsymbol{\gamma}, \mathbf{e}, \boldsymbol{\kappa}, \mathbf{m}, \mathbf{t}, \mathbf{u}] \in K$, and for each fixed $t \in(-\infty, \infty)$ define the functional $\Phi_{t}\{\cdot\}$ on $K$ by

$$
\begin{aligned}
& \Phi_{i}\{Q\}=\int_{V}\left\{\frac{1}{2} L_{k i \text { imna }} * d \kappa_{n a m} * d \kappa_{j i k}-\frac{1}{2} C_{i i k n} * d\left(\gamma_{k n}-e_{k n}\right) * d\left(\gamma_{i i}-e_{j i}\right)\right. \\
& \left.+\frac{1}{2} B_{i k n} * d \gamma_{k n} * d \gamma_{j i}+A_{i i k n} * d e_{k n} * d e_{i i}\right\} d V \\
& -\int_{V}\left\{\rho f_{i} * d u_{i}+\rho b_{i i} * d \psi_{i i}\right\} d V-\int_{S_{i}} \hat{t}_{i} * d u_{i} d S-\int_{S_{\mu}} \hat{\mu}_{i i} * d \psi_{i i} d S .
\end{aligned}
$$

Then

$$
\delta \Phi_{t}\{Q\}=0 \text { over } K,-\infty<t<\infty
$$

if and only if $a$ is a regular solution of $R$.

Proof. Let $\tilde{\boldsymbol{a}}=[\tilde{\mathbf{u}}, \tilde{\mathbf{\psi}}, \tilde{\boldsymbol{\gamma}}, \tilde{\mathbf{e}}, \tilde{\mathbf{k}}, \tilde{\mathbf{m}}, \tilde{\mathbf{t}}, \tilde{\mathbf{u}}]$ be an admissible state and suppose that

$$
Q+\alpha \tilde{a} \in K \text { for every scalar } \alpha \text {. }
$$


Since $K$ is the set of all kinematically admissible states, (4.13) implies $\tilde{Q}$ meets (3.1), (3.4)-(3.6) with

$$
\begin{array}{ccc}
\tilde{u}_{i}=0 & \text { on } & S_{u} x(-\infty, \infty), \\
\tilde{\psi}_{i i}=0 & \text { on } & S_{\psi} x(-\infty, \infty) .
\end{array}
$$

Now use (3.1), (3.4)-(3.6), (3.15), (3.11), the divergence theorem, property (ii) of admissible states and Theorems 1.2 and 1.6 of [9] to verify that

$$
\begin{aligned}
\delta_{Q}^{\tilde{Q}} \Phi_{t}\{Q\}= & \int_{S_{t}}\left(t_{i}-\hat{t}_{i}\right) * d \tilde{u}_{i} d S+\int_{S_{\mu}}\left(\mu_{i j}-\hat{\mu}_{i j}\right) * d \tilde{\psi}_{i j} d S \\
& -\int_{V}\left\{\left(t_{i, i}+\rho f_{i}\right) * d \tilde{u}_{i}+\left(t_{i i}-m_{i i}+\mu_{k i, k}+\rho b_{i i}\right) * d \tilde{\psi}_{j i}\right\} d V
\end{aligned}
$$

for every $\tilde{\mathbf{u}}, \tilde{\mathbf{u}}$ which meet (4.14) and property (i) of admissible states. Clearly (4.15) implies (4.12) if $Q$ is a regular solution of $R$.

Turning to the "only if" portion of the proof, we assume (4.12) holds and choose $\tilde{\mathbf{u}}(\mathbf{x}, t), \tilde{\mathbf{\psi}}(\mathbf{x}, t)$ as in (4.5), where now (since (4.1) holds) $\mathbf{u}^{\prime}$ and $\boldsymbol{\psi}^{\prime}$ are twice continuously differentiable on $\bar{V}$ and

$$
\mathbf{u}^{\prime}=0 \text { on } S_{u}, \quad \boldsymbol{\psi}^{\prime}=0 \text { on } S_{\psi} .
$$

Then by (4.12) and (4.15)

$$
\begin{aligned}
\int_{S,}\left(t_{i}-\hat{t}_{i}\right) u_{i}^{\prime} d S+\int_{S_{\mu}}\left(\mu_{i j}-\hat{\mu}_{i j}\right) \psi_{i j}^{\prime} d S \\
-\int_{V}\left\{\left(t_{i i, i}+\rho f_{i}\right) u_{i}^{\prime}+\left(t_{i i}-m_{i i}+\mu_{k i, k}+\rho b_{i i}\right) \psi_{j i}^{\prime}\right\} d V=0
\end{aligned}
$$

on $-\infty<t<\infty$ for every $\mathfrak{u}^{\prime}, \boldsymbol{\psi}^{\prime}$ with the foregoing properties. This fact, by virtue of the fundamental lemma of the calculus of variations, implies that $Q$ meets (3.2), (3.3), (3.9 $),\left(3.9_{2}\right)$; hence, $Q$ is a regular solution and the proof is complete.

\section{ReFEREnCES}

[1] M. E. Gurtin, Variational principles in the linear theory of viscoelasticity, Arch. Rat. Mech. Anal. 13, 179-191 (1963)

[2] Hu Hai-Chang, On some variational principles in the theory of elasticity and the theory of plasticity, Sci. Sinica 4, 33-54 (1955)

[3] K. Washizu, On the variational principles of elasticity and plasticity, ASRL TR 25-18, Mass. Inst. Tech., Cambridge, Mass., 1955

[4] R. D. Mindlin, Microstructure in linear elasticity, Arch. Rat. Mech. Anal. 16, 51-78 (1964)

[5] R. A. Toupin, Theories of elasticity with couple-stress, Arch. Rat. Mech. Anal. 17, 85-112 (1964)

[6] E. S. Suhubi and A. C. Eringen, Nonlinear theory of microelastic solids. II, Internat. J. Engrg. Sci. 2, 389-404 (1964)

[7] N. Fox, A continuum theory of dislocations for polar elastic materials, Quart. J. Mech. Appl. Math. $19,343(1966)$

[8] A. E. Green, P. M. Naghdi and R. S. Rivlin, Directors and multipolar displacements in continuum mechanics, Internat. J. Engrg. Sci. 2, 611-620 (1965)

[9] M. E. Gurtin and E. Sternberg, On the linear theory of viscoclasticity, Arch. Rat. Mech. Anal. 11, 291-356 (1962)

[10] C. N. DeSilva and K. A. Kline, Nonlinear constitutive equations for directed viscoelastic materials with memory, Z. Angew. Math. Phys. 19, 128-139 (1968) 
[11] K. A. Kline, On thermodynamics of directed continuous media, Z. Angew. Math. Phys. 19, 793-802 (1968)

[12] J. L. Ericksen and C. Truesdell, Exact theory of stress and strain in rods and shells, Arch. Rat. Mech. Anal. 1, 295-323 (1958)

[13] W. T. Koiter, Couple-stresses in the theory of elasticity. II, Nederl. Akad. Wetensch. Proc. Ser. B 67, 30-44 (1964)

[14] P. M. Naghdi, On a variational theorem in elasticity and its application to shell theory, Trans. ASME Ser. F, J. Appl. Mech. 31, 647-653 (1964)

[15] W. Nowacki, Couple-stresses in the theory of thermoelasticity. II, Bull. Acad. Polon. Sci. Sér. Sci. Tech. 14, 203-212 (1966)

[16] E. Reissner, On the foundations of the theory of elastic shells, Proc. 11th Internat. Congr. of Appl. Mech. (Munich, 1964), Springer-Verlag, Berlin, 1966

[17] W. Günther, Zur Statik und Kinematik des Cosseratschen Kontinuums, Abh. Braunschweig. Wiss. Ges. 10, 195-213 (1958)

[18] W. Nowacki, Couple-stresses in the theory of thermoelasticily. III, Bull. Acad. Polon. Sci. Sér. Sci. Tech. 14, 505-513 (1966)

[19] E. Reissner and F. Y. M. Wan, A note on Günther's analysis of couple stress, Proc. IUTAM Sympos. (1967), Mechanics of generalized continua, Springer-Verlag, Berlin, 1968

[20] E. Reissner, A note on variational principles in elasticity, Internat. J. Solids Structures 1, 93 (1965)

[21] J. L. Ericksen, Anisotropic fluids, Arch. Rat. Mech. Anal. 4, 231-237 (1960)

[22] K. A. Kline, S. J. Allen and C. N. DeSilva, A continuum approach to blood flow, Biorheology 5, $111-118(1968)$

[23] O. D. Kellogg, Foundations of potential theory, Dover, New York, 1953

[24] S. J. Allen, C. N. DeSilva and K. A. Kline, Theory of simple deformable directed fluids, Phys. Fluids 10, 2551-2555 (1967)

[25] A. E. Green and R. S. Rivlin, On Cauchy's equations of motion, Z. Angew. Math. Phys. 15, 290292 (1964) 